\title{
Controlling the Dynamics and Stabilization of the Altitude and Attitude of An Underactuated Tri-rotor UAV
}

\author{
Zain Anwar Ali ${ }^{1}$, Muhammad Aamir ${ }^{2}$, Dao Bo Wang ${ }^{1}$, Muhammad Anwar \\ Ahmed $^{2}$, and Suhaib Masroor ${ }^{3}$ \\ ${ }^{1}$ College of Automation Engineering, Nanjing University of Aeronautics \& Astronautics, \\ Nanjing, Jiangsu, China \\ ${ }^{2}$ Electronic Engineering Department, Sir Syed University of Engineering \& Technology, \\ Karachi, Pakistan \\ ${ }^{3}$ School of Mechatronic \& Automation Engineering, Shanghai University, Shanghai, \\ China \\ Email: zainanwar86@hotmail.com,dbwangpe@nuaa.edu.cn,muaamir5@yahoo.com, \\ engr_anwar_ahmed@outlook.com, suhaibmasroor1@gmail.com.
}

\begin{abstract}
In this article, an Adaptive Sliding Mode Control (ASMC) method is proposed for controlling the dynamics and stabilization of the altitude and attitude of an underactuated tri-rotor Unmanned Aerial Vehicle (UAV). The model of UAV is highly nonlinear and complex in nature with the six degree of freedom (6-DOF) and subdivided in to fully actuated and underactuated subsystems which is controlled by the sliding surface of ASMC. Furthermore, the stability of a system is validated using Lyapunov stability theory. Simulation results showed that the proposed control scheme enabled the tri-rotor UAV to fly effectively when compared with Adaptive Robust Backstepping (ARB) control. Lastly, an external disturbance is added in the system to check the effectiveness of the proposed scheme in which a wind disturbance test is done with the proposed controller that shows the robustness, fast error convergence and good transient behavior.
\end{abstract}

Keywords: Tri-rotor UAV, underactuated system, Adaptive Sliding Mode Control $(A S M C)$

\section{Introduction}

Unmanned Aerial Vehicle (UAV) is the most remarkable inventions in the 21st century [1]. The UAV is divided in to two categories, one is fixed wing and second is rotor craft UAV's. However, rotor craft UAV's have an advantage it does not need a runway to execute a flight because of its Vertical Takeoff and Landing (VTOL) ability [2-4]. Furthermore, the rotor craft UAV's are also classified in to bi-rotor, tri-rotor, quad-rotor and hex-rotor crafts, having Multiple Input and Multiple Output (MIMO) underactuated systems. These days the usage of UAV is quite common in our society, they are commonly used in surveillance, rescue and searching tasks and etc. [5-7].

Due to its compact size and VTOL ability, it hovers with very simple construction and maintenance. A rotor craft has four input control commands called as altitude, lateral, longitudinal and angular moments (Col., Lon., Lat., Ped.,). The linear and angular velocity subcomponents are $(\mathrm{u}, \mathrm{v}, \mathrm{w})$ and $(\mathrm{p}, \mathrm{q}, \mathrm{r}) .(\varphi, \theta, \psi)$ are the Euler angles of the aerial vehicle. A number of physical effects that UAV can exhibit includes inertial torque, effects of the gyroscope and aerodynamics forces making it difficult for UAV to perform the successful flight operation in the real world [8].

In this article, the model of tri-rotor UAV is used due to its properties of strongly coupled, multivariable, nonlinear, and underactuated in nature along with (6-DOF) the six degree of freedom [9-11]. To establish the nonlinear control scheme to control the model 
of 6-DOF organize with linear and angular velocities. The enquiry phase directs a fault which is established in the moment of yaw control induced due to the reaction and torque which is produced by the rotor. To fix, this fault, a series of mechanical designs of trirotor UAV's has been prepared to their own parameters. After, that a Brushless Direct Current (BLDC) motor is mounted, which tilt the yaw control to nullify the moments [12]. The key role of this mechanical design is better turning, movement by utilizing the tilt rotor axes.

Before the real flight simulation, to simulate the nonlinear model of tri-rotor UAV in this article, its Euler angles, linear and angular velocity subcomponents, control commands and track the reference path. The dynamic model of the UAV is highly coupled with a lot of parameter uncertainties to which a prospective controller has to be robust [13-15]. While, the efficiency and performance will possibly be reduced in the situation of a collapse, it requires that the controller must stabilize the system and bears compact functions, in which steady hovering and safe flight is done.

Formerly, different control algorithms were used to control the dynamics of underactuated UAV. PID, the most classical method that was used in [16], backstepping control of underactuated system used in [17], Sliding Mode Control (SMC) was already used in [18]. In this article, we used an Adaptive Sliding Mode Controller (ASMC) along with the wind disturbance test, which was not used in any of the aforementioned work.

To validate the effectiveness of our proposed ASMC, it is compared with Adaptive Robust Backstepping (ARB) Controller of [19] without disturbance and with the wind disturbance test to cross check the efficiency and robustness.

The key contributions of this article are: (1) an adaptive sliding surface is proposed to remove the steady state error and for the rejections of model uncertainties (2) the designed ASMC uses linear and angular velocity subcomponents as an input providing practical inside of the real world scenario (3) in spite of the model uncertainties, close loop errors in the UAV system converges to zero, which is proved in the simulation and result section (4) stability of the system is proven by Lyapunov stability function.

The breakup of the article is structured as follows. Section 2 provides the preliminaries about tri-rotor UAV, which is followed by its dynamics while problem is described in Section 3. Section 4 discuss the control algorithm and Section 5 defines the simulation and result analysis. Finally, Section 6 concludes the whole article.

\section{The Preliminaries of Tri-Rotor UAV}

Figure 1(a) describe the 2-D view of UAV, while Figure 1(b) defines the 3-D view of the aerial robot. 


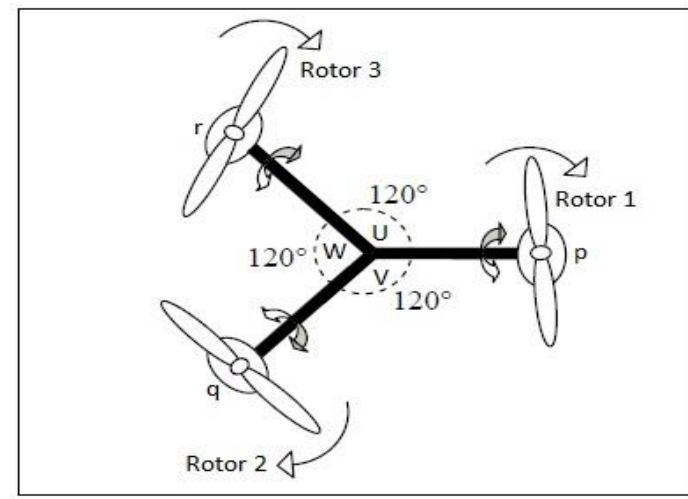

(a)

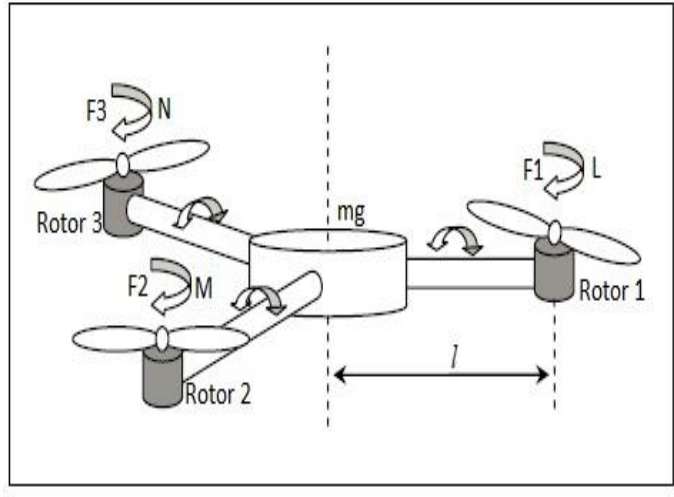

(b)

Figure 1 (a). The Upper Vision of UAV along with Angular and Linear Velocity Rates (b): The 3-D Vision of UAV with Respect to the Center of Gravity

The mathematical model can be written as,

$$
\left\{\begin{array}{c}
m u=m g-T \\
I=W(T) \delta \\
j \delta=\delta * j \delta+\tau
\end{array}\right.
$$

In the above equation (01) $v \in R$, the velocity of the UAV is dependent on its rotational matrix "R" which defines the position of the vehicle. The angular velocity " $\delta$ " of the vector coordinates is also dependent on the rotational matrix [20-22]. Where " $m$ " is the mass and $\mathrm{j} \in R$ inertial points, " $T$ " is the rotational torque, $\tau \in R$. Now the Euler angles $\Gamma=(\varphi, \theta, \psi)$ is used to derive the attitude of the UAV around $\mathrm{x}, \mathrm{y}, \mathrm{z}$ axes. Now the movement around $\mathrm{x}, \mathrm{y}, \mathrm{z}$ axis are designated as roll, pitch and yaw respectively [23] [24]. Now the rotation matrix can be written as,

$$
\left\{\begin{array}{c}
W(T)=\left[\begin{array}{ccc}
1 & 0 & 0 \\
0 & \cos \varphi & \sin \varphi \\
0 & -\sin \varphi & \cos \varphi
\end{array}\right]\left[\begin{array}{ccc}
\cos \theta & 0 & -\sin \theta \\
0 & 1 & 0 \\
\sin \theta & 0 & \cos \theta
\end{array}\right]\left[\begin{array}{ccc}
\cos \psi & \sin \psi & 0 \\
-\sin \psi & \cos \psi & 0 \\
0 & 0 & 1
\end{array}\right] \\
\cos \theta \cos \psi \\
R(T)=\left[\begin{array}{ccc}
\cos \theta \sin \psi & -\sin \theta \\
\sin \varphi \sin \theta \cos \psi-\cos \psi \sin \psi & \sin \varphi \sin \theta \sin \psi-\cos \varphi \cos \psi & \sin \varphi \cos \theta \\
\cos \varphi \cos \theta \cos \psi+\sin \varphi \sin \psi & \cos \varphi \sin \theta \sin \psi-\sin \theta \cos \psi & \cos \psi \cos \theta
\end{array}\right]
\end{array}\right.
$$

The tri-rotor aerial vehicle has three motors which is fixed in its triangular frame of the body, the input and output force and torque is written as,

$$
\left\{\begin{array}{c}
f_{i}=k t u_{i}^{2} \rightarrow k t u_{i}\left|u_{i}\right| \\
\tau_{i}=k \tau u_{i}^{2} \rightarrow k \tau u_{i}\left|u_{i}\right|
\end{array}\right.
$$

Where $\mathrm{i}=1,2,3$ for all three rotors of the aerial vehicle.

The tri-rotor UAV system is a combination of cascades and the complex dynamics of the underactuated system. Due to their complex coupling relationship exists in the system, the design and stability analysis of the vehicle is also quite complex [25]. Initially, the system model decoupled into fully actuated subsystem and an underactuated subsystem. Thus by simplifying the controller design and its stability analysis by using Lyapunov stability analysis. Now according to the principle of rotor craft UAV, adjusting the rotational speed of the motor controls the attitude of the vehicle [26]. By changing the rotor speed the direction of the vehicle can be controlled. Now with respect to Newton's second law, the sum of external forces acted on the aircraft is equivalent to the frame of time that changes the body momentum. Additionally, external moments acting on the UAV is equivalent to change the rate of rotational movements [27-29].

Translational and rotational moment variation is referred as an inertial reference frame. The UAV aircraft has a proficiency of identical forces, aerodynamic moments and velocity components which can make into a six-degree of freedom (6-DOF) equation of 
motion those are nonlinear in nature [30]. Now, according to the rotors of the aircraft, to get the vehicle to keep in hover the basic speed of the motor taking into account that the aircraft is in hovering state. The total force in the aircraft exerted on the body which can be expressed as,

$$
\sum_{\mathrm{i}}^{\mathrm{g}} F_{\mathrm{i}}=m g
$$

\section{Dynamics of Tri-Rotor UAV \& Its Problem Description}

The dynamic equations of the aircraft is defined in the equation (05) to (12) which are described below. The linear, angular velocity components and Euler angles are defined by (p, q, r), (u, v, w) and $(\varphi, \theta, \psi)$. Therefore, the control variables are described as follows: $\left(\nabla \delta_{T}, \nabla \delta_{\varphi}, \nabla \delta_{\theta}, \nabla \delta_{\psi}\right)$, respectively for the basic speed of the aircraft to maintain hovering [31].

Linear system dynamics are,

$$
\left\{\begin{array}{c}
\dot{u}=(1 / m)\left(F_{1} \cos \theta \cos \psi+F_{2} \cos \theta \sin \psi-F_{3} \sin \theta\right) \\
\dot{v}=(1 / m)\left(F_{1}(\sin \varphi \sin \theta \cos \psi-\cos \varphi \sin \psi)+F_{2}(\sin \varphi \sin \theta \sin \psi+\cos \theta \cos \psi)+F_{1}(\sin \varphi \cos \theta)\right)
\end{array}\right.
$$

$\dot{w}=(1 / m)\left(F_{1}(\cos \varphi \sin \theta \cos \psi+s i c\right.$
Angular system dynamics are,

$$
\left\{\begin{array}{c}
\dot{p}=\left(I_{y}-I_{z} / I_{x}\right)(r * q)+\left(1 / I_{x}\right)(\sqrt{3} * L / 2)\left(F_{2}-F_{a}\right) \\
\dot{q}=\left(I_{z}-I_{x} / I_{y}\right)(r * p)+\left(1 / I_{y}\right)(1 / 2)\left(F_{2}+F_{2}\right)-L * f_{1} \cos \nabla \delta_{\psi}+\tau_{1} \sin \nabla \delta_{\psi} \\
\dot{r}=\left(I_{x}-I_{y} / I_{z}\right)(q * p)+\left(1 / I_{z}\right)(1 / 2)\left(F_{2}+F_{a}\right) L * f_{1} \sin \nabla \delta_{\psi}-\tau_{1} \cos \nabla \delta_{\psi}-\tau_{2}-\tau_{a}
\end{array}\right.
$$

According to the definition of tri-rotor the speed control of three motors and its motion relationship can be written as,

$$
\left\{\begin{array}{l}
F_{1}=C_{\pi} \rho A r^{2}\left(\delta_{T}+\nabla \delta_{T}-\nabla \delta_{\theta}+\nabla \delta_{\psi}\right)^{2} \\
F_{2}=C_{\pi} \rho A r^{2}\left(\delta_{T}+\nabla \delta_{T}+\nabla \delta_{\varphi}-\nabla \delta_{\psi}\right)^{2} \\
F_{2}=C_{\pi} \rho A r^{2}\left(\delta_{T}+\nabla \delta_{T}-\nabla \delta_{\theta}+\nabla \delta_{\psi}\right)^{2}
\end{array}\right.
$$

Table 1. Tri-Rotor UAV Dynamics \& Its Constants

\begin{tabular}{|c|c|c|c|}
\hline $\begin{array}{c}\text { Axis } \\
\text { system } \\
(\mathrm{x}, \mathrm{y}, \mathrm{z})\end{array}$ & $\begin{array}{c}\text { Linear } \\
\text { velocity } \\
\text { components }\end{array}$ & $\begin{array}{c}\text { Rotational } \\
\text { velocity } \\
\text { components }\end{array}$ & $\begin{array}{c}\text { Inertial } \\
\text { Coordinates }\end{array}$ \\
\hline Roll $_{(\varphi)}$ & $\mathrm{u}$ & $\mathrm{p}$ & $\mathrm{I}_{\mathrm{x}}$ \\
\hline Pitch $_{(\theta)}$ & $\mathrm{v}$ & $\mathrm{q}$ & $\mathrm{I}_{\mathrm{y}}$ \\
\hline Yaw $_{(\psi)}$ & $\mathrm{w}$ & $\mathrm{r}$ & $\mathrm{I}_{\mathrm{z}}$ \\
\hline
\end{tabular}

Hovering: The state of hover or hovering of tri-rotor is based on the blades of the propeller which increases the blade numbers on the propeller and restricts the speed of forward flight. Now employing the theory of momentum for the dynamics of helicopter which is also related to the tri-rotor UAV theory [32]. Whereas, " $C_{\pi}$ " is the coefficient of thrust, " $\rho$ " is the density of air, " $A$ " is the blade area, " $r$ " is the blade radius, " $\delta_{T}$ " is the angular velocity of thrust and " $C_{Q}$ " is the rotor shaft constant.

Case for hovering: If the aircraft is hovering the following conditions must be true. (1): $\varphi=\dot{\varphi}=0,(2): \theta=\dot{\theta}=0,(3): \psi=\dot{\psi}=0$ and (4): $\operatorname{Cos} \varphi=\operatorname{Cos} \theta=1$. The tri-rotor UAV flight in a hover state in the equilibrium point condition at that time by compared with $\delta_{T}$ than $\nabla \delta_{T}, \nabla \delta_{\varphi}, \nabla \delta_{\theta}, \nabla \delta_{\psi}$ are high order small qualities so $3 C_{\pi} \rho A r^{2} \delta_{T}^{2}=m g$, than we get: $\delta_{T}=\left(m g / 3 C_{\pi} \rho A r^{2}\right)^{1 / 2}$.

The dynamics of the tri-rotor UAV is obtained as follows, 


$$
\left\{\begin{array}{c}
\ddot{x}=(1 / m)(\cos \varphi \cos \psi \sin \theta+\sin \psi \sin \varphi) \sum_{i}^{3} F_{i} \\
\ddot{y}=(1 / m)(\sin \theta \sin \psi \cos \varphi-\sin \varphi \cos \psi) \sum_{i}^{3} F_{i} \\
\ddot{z}=-g+(1 / m)(\cos \theta \cos \varphi) \sum_{i}^{3} F_{i}
\end{array}\right.
$$

By using equation (5) and (7) and taking into account the effect of high-order small quantities which can be expressed as $\sum_{i}^{3} F_{i}=m g+8 C_{\pi} \rho A r^{2} \delta_{T} \nabla \delta_{T}$ and then after getting the location of subsystem simplification system dynamics equations are ,

$$
\left\{\begin{array}{c}
\ddot{x}=g(\theta \operatorname{Cos} \psi+\varphi \sin \psi) \\
\ddot{y}=g(\theta \sin \psi-\varphi \cos \psi) \\
\ddot{z}=8 C_{\pi} \rho A r^{2} \delta_{T} \nabla \delta_{T} / m
\end{array}\right.
$$

The attitude kinematics equations of tri-rotor UAV is obtained as,

$$
\left\{\begin{array}{c}
\delta_{x}=\dot{\varphi} \operatorname{Cos} \theta-\dot{\psi} \operatorname{Cos} \varphi \operatorname{Sin} \theta \\
\delta_{y}=\dot{\theta}+\dot{\psi} \operatorname{Sin} \varphi \\
\delta_{z}=\dot{\varphi} \operatorname{Sin} \theta+\dot{\psi} \operatorname{Cos} \theta \operatorname{Cos} \varphi
\end{array}\right.
$$

The attitude dynamic equations of tri-rotor UAV are,

$$
\left\{\begin{array}{l}
I_{x} \dot{\delta}_{x}=C_{\pi} \rho A r^{2} d+\left(I_{y}-I_{z}\right) \delta_{y} \delta_{z} \\
I_{y} \delta_{y}=C_{\pi} \rho A r^{2} d+\left(I_{z}-I_{x}\right) \delta_{z} \delta_{x} \\
I_{z} \delta_{z}=C_{\pi} \rho A r^{2} d+\left(I_{x}-I_{y}\right) \delta_{x} \delta_{y}
\end{array}\right.
$$

Let us suppose that the motion model of the system can be simplified as,

$$
\begin{gathered}
\left\{\begin{array}{l}
\delta_{x}=\dot{\varphi} \\
\delta_{y}=\dot{\theta} \\
\delta_{z}=\dot{\psi}
\end{array}\right. \\
\left\{\begin{array}{l}
\delta_{x}=3 d C_{\pi} \rho A r^{2} \delta_{T} \nabla \delta_{\varphi} / I_{x} \\
\delta_{y}=3 d C_{\pi} \rho A r^{2} \delta_{T} \nabla \delta_{\theta} / I_{y} \\
\delta_{z}=3 d C_{\pi} \rho A r^{2} \delta_{T} \nabla \delta_{\psi} / I_{z}
\end{array}\right.
\end{gathered}
$$

The model simplification of a tri-rotor UAV system is a typical underactuated system and 6- degree of freedom (6-DOF) via four control inputs typically named as altitude, lateral, longitudinal and angular moments $\left(\nabla \delta_{T}, \nabla \delta_{\varphi}, \nabla \delta_{\theta}, \nabla \delta_{\psi}\right)$ [33-35]. But in fact, in aircraft systems there are two non-holonomic constraints that is:

$$
\left\{\begin{array}{c}
\operatorname{Sin} \varphi=(-\ddot{x} \operatorname{Sin} \varphi+\ddot{y} \operatorname{Cos} \varphi) /\left(\ddot{x}^{2}+\ddot{y}^{2}+(\ddot{z}+g)\right)^{1 / 2} \\
\operatorname{Tan} \theta=(\ddot{x} \operatorname{Cos} \varphi+\ddot{y} \operatorname{Sin} \varphi / \ddot{z}+g)
\end{array}\right.
$$

Due to the presence of two non-holonomic constraints, causes the vehicle to realize the full meaning of six degrees of freedom. Whereas the roll and pitch are attitude which is bounded by the position error of the aircraft [36-37].

$$
\left\{\begin{array}{l}
\ddot{z}=8 C_{\pi} \rho A r^{2} \delta_{T} \nabla \delta_{T} / m \\
\ddot{\psi}=8 C_{Q} \rho A r^{3} \delta_{T} \nabla \delta_{T} / I_{z}
\end{array}\right.
$$

The underactuated system dynamic equations are,

$$
\begin{array}{r}
\left\{\begin{array}{l}
\ddot{x}=g(\theta \operatorname{Cos} \psi+\varphi \sin \psi) \\
\ddot{y}=g(\theta \sin \psi-\varphi \cos \psi)
\end{array}\right. \\
\left\{\begin{array}{l}
\ddot{\varphi}=3 d C_{\pi} \rho A r^{2} \delta_{T} \nabla \delta_{\varphi} / I_{x} \\
\ddot{\theta}=3 d C_{\pi} \rho A r^{2} \delta_{T} \nabla \delta_{\theta} / I_{y}
\end{array}\right.
\end{array}
$$




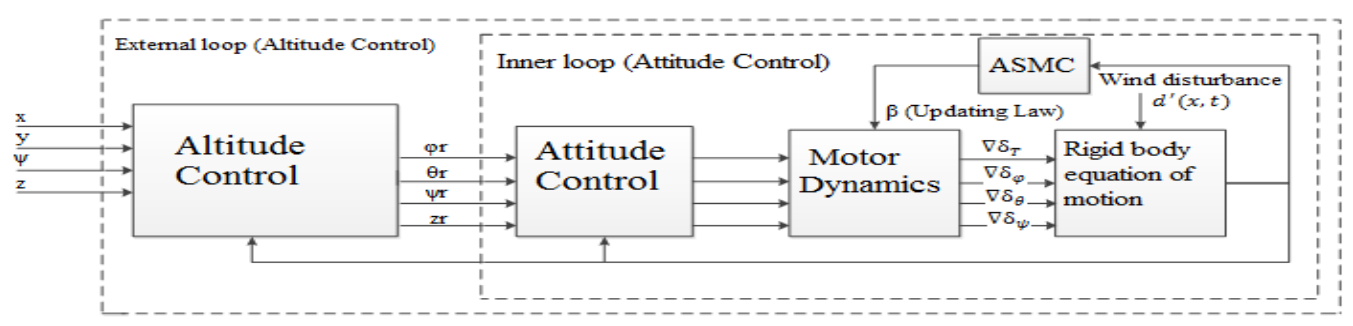

Figure 2. Complete Block Diagram of Control System

\section{Controller Designing}

In this part, of the article the complete hierarchy of ASMC is defined. In which observer is designed for the inner loop to control the attitude of tri-rotor UAV, which is shown in Figure 2. The height or altitude and yaw control equation in the absence of disturbance is written as,

$$
\left\{\begin{array}{c}
\nabla \delta_{T}=\left(m / 8 C_{\pi r} \rho A r^{2} \delta_{T}\right)\left(k_{z 1}\left(z_{d}-z\right)-k_{z 2} \dot{z}+k_{z 3} \int\left(z_{d}-z\right) d t\right) \\
\nabla \delta_{\psi}=\left(I_{z} / 8 C_{Q} \rho A r^{3} \delta_{T}\right)\left(k_{\psi 1}\left(\psi_{d}-\psi\right)-k_{\psi 2} \dot{\psi}+k_{\psi 3} \int\left(\psi_{d}-\psi\right) d t\right)
\end{array}\right.
$$

The design process of the adaptive sliding mode controller for the underactuated subsystem

is presented

below.

Now $x_{1}=\left[\begin{array}{ll}x & y\end{array}\right]^{T}, x_{2}=[\dot{x} \dot{y}]^{T}, x_{3}=[\varphi \theta]^{T}, x_{4}=[\dot{\varphi} \dot{\theta}]^{T}, u=\left[\nabla \delta_{\varphi} \nabla \delta_{\theta}\right]^{T}$, the system can be written as the following standard form:

$$
\begin{aligned}
& \left\{\begin{array}{c}
\dot{x}_{1}=x_{2} \\
\dot{x}_{2}=g_{1}\left(x_{3}\right)+d^{\prime}(x, t) \\
\dot{x}_{3}=x_{4} \\
\dot{x}_{4}=g_{2}(u)+d^{\prime}(x, t)
\end{array}\right. \\
& \text { In which, } \quad g_{1}\left(x_{3}\right)=\left[\begin{array}{cc}
g \operatorname{Sin} \psi & g \operatorname{Cos} \psi \\
-g \operatorname{Cos} \psi & g \operatorname{Sin} \psi
\end{array}\right] x_{3} \\
& g_{2}(u)=\left[\begin{array}{cc}
3 d C_{\pi} \rho A r^{2} \delta_{T} / I_{x} & 0 \\
0 & 3 d C_{\pi} \rho A r^{2} \delta_{T} / I_{y}
\end{array}\right] u,
\end{aligned}
$$

Lemma 1: " $d$ ' $(x, t)$ " is the external disturbance. Now adaptive base SMC error vector can be written as $e_{1}=x_{1}-x_{1 d}, e_{2}=x_{2}, e_{3}=g_{1}\left(x_{3}\right), e_{4}=\left(\partial g_{1}\left(x_{3}\right) x_{4}\right) / \partial x_{3}, E_{r r}=\left[e_{1} e_{2} e_{3}\right]$, the UAV positional control " $x_{1 d}$ " is the constant, then $x_{1}=0$. Defines a system $S=C_{1} e_{1}+C_{2} e_{2}+C_{3} e_{3}+C_{4}$.

Proposition 1: Let $d^{\prime}(x, t)$ is the bounded disturbance and satisfy: $\left\|E_{r r}\right\| \bar{d}^{\prime} \geq \bar{d}^{\prime}$.

Supposition: The adaptive sliding mode variable structure control is obtained, so that the points lie on the sliding surface $S=0$ are the end points which satisfy the following condition: $S=0, \hat{S}=0$, and $\vec{S}=0$. Ensures that the system state is always in the sliding surface when $S=0$, the controller is equivalent to " $u_{\text {eqq }}$ ". Through the position $\dot{S}=0$ the equivalent control of the adaptive sliding mode controller can be obtained as,

$$
u_{e q}=-\left[\partial g_{1}\left(x_{3}\right) g_{2} / \partial x_{3}\right]^{-1}\left[C_{1} x_{2}+C_{2} g_{1}\left(x_{3}\right)+C_{3}\left(\partial g_{1}\left(\partial x_{3}\right) x_{4} / x_{3}\right)\right]
$$

Switching control $u_{\text {swc }}$, can make the adaptive sliding control function uniform "S" converges to zero.

$$
u_{s w c}=-\left[\partial g_{1}\left(x_{3}\right) g_{2} / \partial x_{3}\right]^{-1}[\beta \operatorname{sgn}(S)+\eta \mathrm{S}]
$$

Proposition 2: The updating law of adaptive sliding mode control is $\beta=\left(C_{2} \bar{d}^{\prime}\left\|E_{r r}\right\|+\sqrt{2} * g \bar{d}^{\prime}\left\|E_{r r}\right\|\right)+\alpha$.

At that time, the adaptive sliding mode controller is obtained as, 
Now, the derivative of $g_{1}\left(x_{3}\right)$ is,

$$
u=u_{e q}+u_{s w c}
$$

$$
\frac{\partial g_{1}\left(x_{g}\right)}{\partial x_{\mathrm{a}}}=\left[\begin{array}{cc}
g \operatorname{Sin} \psi & g \operatorname{Cos} \psi \\
-g \operatorname{Cos} \psi & g \operatorname{Sin} \psi
\end{array}\right]
$$

$\left\|\partial g_{1}\left(x_{3}\right) / \partial x_{3}\right\|=\sqrt{2} * g$, which states that $\sqrt{2} * g \bar{d}^{\prime}\left\|E_{r r}\right\| \geq \mathrm{d}^{\prime} \geq\left\|\partial g_{1}\left(x_{3}\right) / \partial x_{3}\right\|$.

Proof: The stability of the UAV system is defined by using Lyapunov candidate function which is, $V=(1 / 2) S^{T} S$

Invoking the lemma (1) and equation (23) than the Lyapunov function derivative with respect to time is written as,

$$
\vec{V}=\|S\| \alpha-\eta S^{T} S \geq S^{T}\left[C_{1} e_{2}+C_{2}\left(e_{3}+d^{\prime}\right)+C_{3} e_{4}+\partial g_{1}\left(x_{3}\right) / \partial x_{3}\left(g_{2}(u)+d^{\prime}(x, t)\right)\right]
$$

The derivative of Lyapunov candidate function is negative with respect to time, therefore the adaptive sliding mode function " $S$ " converges to a zero, in the infinite state of time. In this case, the system can be reduced to an agitation system as follows:

$$
\begin{aligned}
& \dot{E}_{r r}=\mathrm{d}^{\prime}(x, t)+A E_{r r} \\
& A=\left[\begin{array}{ccc}
0 & I_{2} & 0 \\
0 & 0 & I_{2} \\
-C_{1} I_{2} & -C_{1} I_{2} & -C_{1} I_{2}
\end{array}\right], d=\left[\begin{array}{c}
0 \\
d^{\prime} \\
0
\end{array}\right],\left\|E_{r r}\right\| \bar{d}^{\prime} \geq\|d\| \text {. Where " } A \text { " is a Hurwitz }
\end{aligned}
$$
matrix.

So for the given condition, $Q=Q^{T}>0$. there is a unique solution for this $P=P^{T}>0$, for Lyapunov stability. Now define a Lyapunov function:

$$
\dot{V}_{1} \leq-\eta_{\min }(Q)\left\|E_{r r}\right\|^{2}+2 \eta_{\max }(P) \bar{d}^{\prime}\left\|E_{r r}\right\|
$$

Lemma 2: As long as inequality $\eta_{\min }(Q) / 2 \eta_{\max }(P)>\bar{d}^{r}$, holds then $V_{1} \leq 0$, the system is asymptotically stable. The system errors $e_{1}, e_{2}, e_{3}$ will converge to zero. According to $S=C_{1} e_{1}+C_{2} e_{2}+C_{3} e_{3}+e_{4}=0$, we knew that system will converge to zero.

\section{Simulation and Result Analysis}

In this part of the article, tri-rotor dynamic model equations (05) to (08) are used to prove the effectiveness and stability of our proposed adaptive sliding mode controller scheme with wind disturbance. Furthermore, the efficiency of the proposed Adaptive Sliding Mode Controller (ASMC) is compared with the Adaptive Robust Back-stepping (ARB) control method for the underactuated UAV [19]. According to the model of the trirotor aerial vehicle, the designing parameters, set the following simulation conditions, in which UAV model parameters shown in the Table 2. 


\section{Table 2. The Tri-Rotor UAV Model Parameters}

\begin{tabular}{|c|c|c|}
\hline Variables & Value & S.I Units \\
\hline (Mass) “m” & 0.75 & $\mathrm{~kg}$ \\
\hline (G.F) “g” & 9.8 & $\mathrm{~m} / \mathrm{s}^{2}$ \\
\hline $\mathrm{L}$ & 0.35 & $\mathrm{M}$ \\
\hline $\mathrm{I}_{\mathrm{x}}$ & 0.41 & $\mathrm{Kgm}^{2}$ \\
\hline $\mathrm{I}_{\mathrm{y}}$ & 0.41 & $\mathrm{Kgm}^{2}$ \\
\hline $\mathrm{I}_{\mathrm{z}}$ & 0.73 & $\mathrm{Kgm}^{2}$ \\
\hline$k_{z 1}$ & 1.66 & $\mathrm{Ns} / \mathrm{m}$ \\
\hline$k_{z 2}$ & 0.33 & $\mathrm{Ns} / \mathrm{m}$ \\
\hline$k_{z 3}$ & 0.33 & $\mathrm{Ns} / \mathrm{m}$ \\
\hline$k_{\psi 1}$ & 1.66 & $\mathrm{Ns} / \mathrm{m}$ \\
\hline$k_{\psi 2}$ & 1 & $\mathrm{Ns} / \mathrm{m}$ \\
\hline$k_{\psi 3}$ & 0.01 & $\mathrm{Ns} / \mathrm{m}$ \\
\hline
\end{tabular}

The control system block diagram is shown in Figure 2, having reference or the desired values of the UAV. The system performance have inner and outer loops such that ASMC control both the loops. Given values using as an input which is constantly changes the position and attitude of UAV by using its linear and angular velocities in the absence of disturbance in simulation case I and in the presence of wind disturbance test in case II, that is exposed to be real application in the real world. In order to achieve required altitude, angular velocity is used to control the error while linear velocity along with angular velocity is used to control the position. The control parameters for the underactuated UAV system is shown in the Table 3.

\section{Table 3. The Tri-Rotor UAV Controller Parameters}

\begin{tabular}{|c|c|}
\hline Variables & Values \\
\hline$C_{1}$ & 10 \\
\hline$C_{2}$ & 11 \\
\hline$C_{3}$ & 4 \\
\hline$\dot{\eta}$ & 0.1 \\
\hline$\beta$ & 0.1 \\
\hline$\rho$ & $1 / \mathrm{kg} \cdot \mathrm{m}^{-3}$ \\
\hline $\mathrm{A}$ & $0.652 / \mathrm{m}^{2}$ \\
\hline$\alpha$ & 0.1 \\
\hline $\mathrm{r}$ & $0.125 / \mathrm{m}^{2}$ \\
\hline$C_{\pi}$ & 0.00451 \\
\hline$C_{Q}$ & 0.000212 \\
\hline $\mathrm{d}$ & $0.35 / \mathrm{m}^{2}$ \\
\hline
\end{tabular}

Case I: In this case of simulation, the comparison of ARB with ASMC is presented without disturbance. Moreover, the simulated results are shown in Figure 3 to 8 . The starting position of UAV is $[0,0,0] \mathrm{m}$, initial attitudes are $[0,0.45,0] \mathrm{rad}$ the reference altitude and attitude in this case are $[10,10,10] \mathrm{m}$ and $[0,0,0] \mathrm{rad}$ which is shown in Figure 3 and 4 respectively. Now by comparing our proposed controller with the ARB controller shows that the ASMC controller has better convergence rate and good transient performance than ARB. Figure 5 and 6 shows the linear and angular velocities subcomponents that converge to zero to fulfill the referred position. Figure 7, shows the input control commands and Figure 8 shows the sliding variables which converge to zero. 

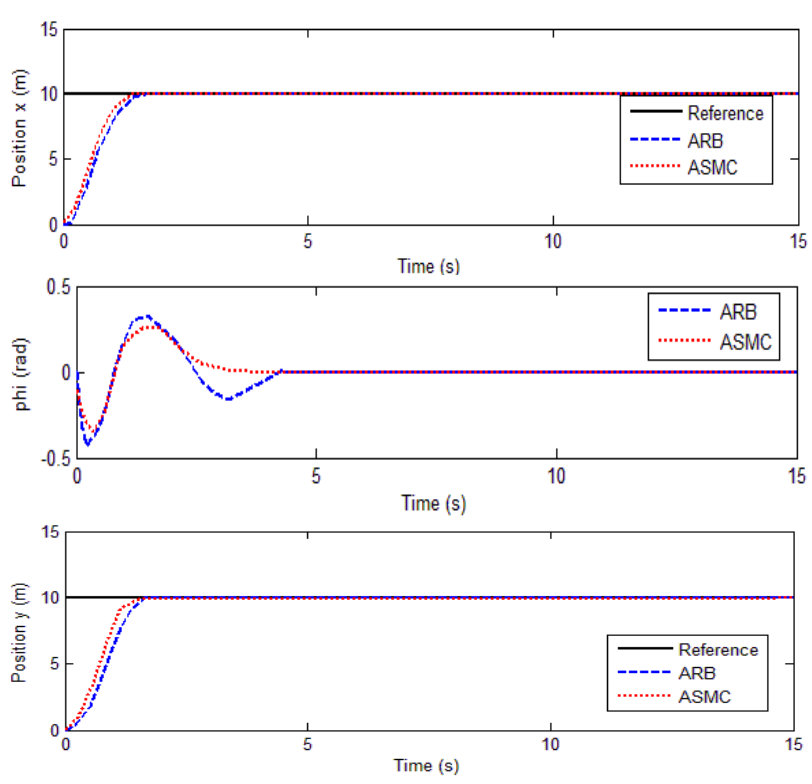

Figure 3. Positions (x, y, z) of UAV
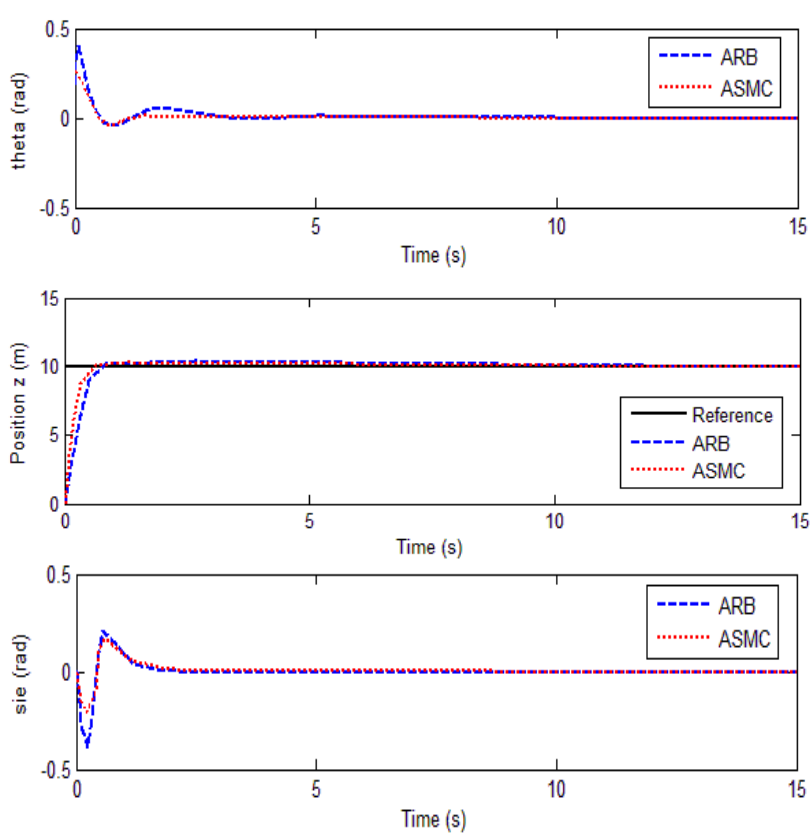

Figure 4. The Euler Angle Responses 

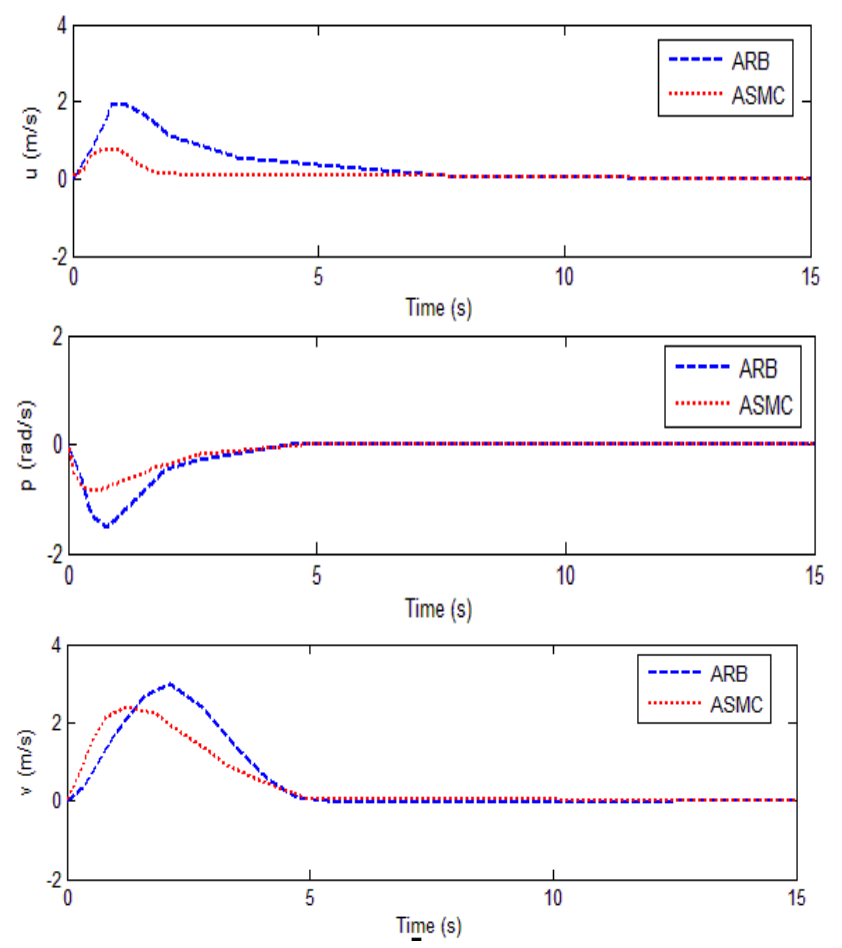

Figure 5. Linear Velocity Components
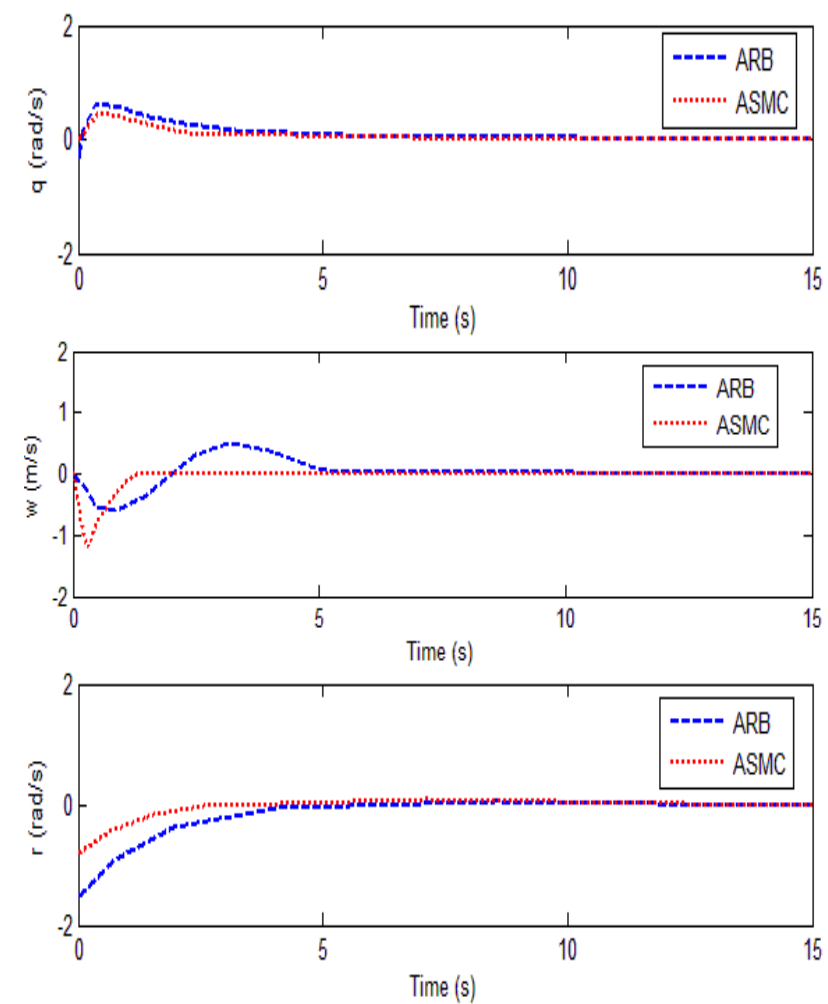

Figure 6. Angular Velocity Components 

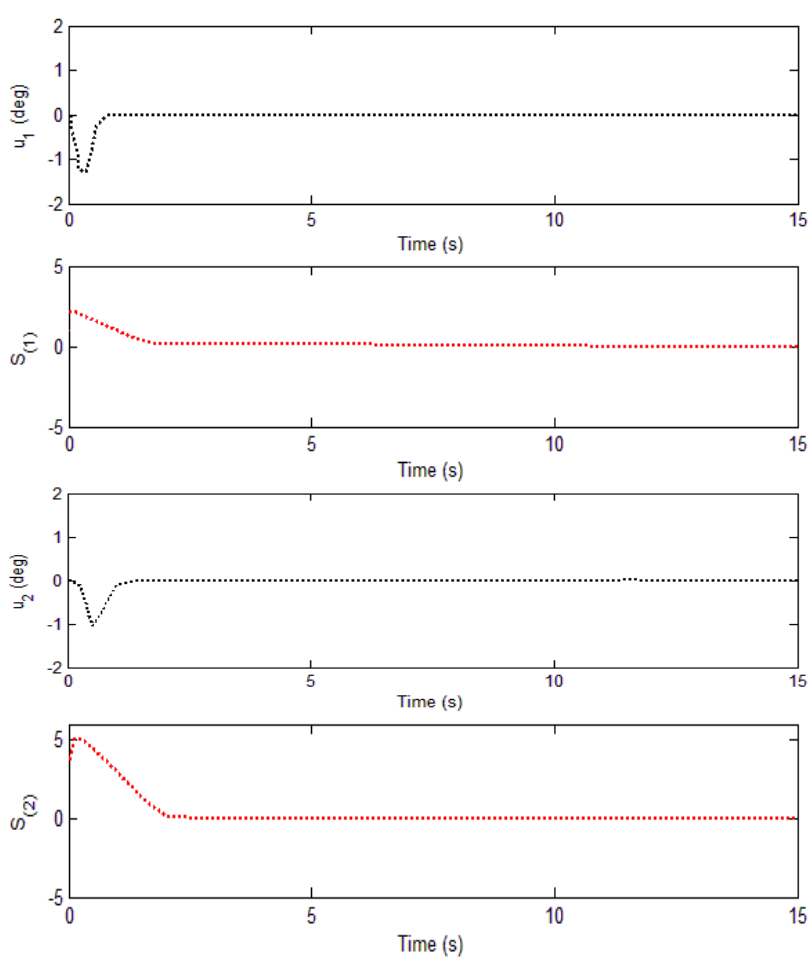

Figure 7. Input Control Commands
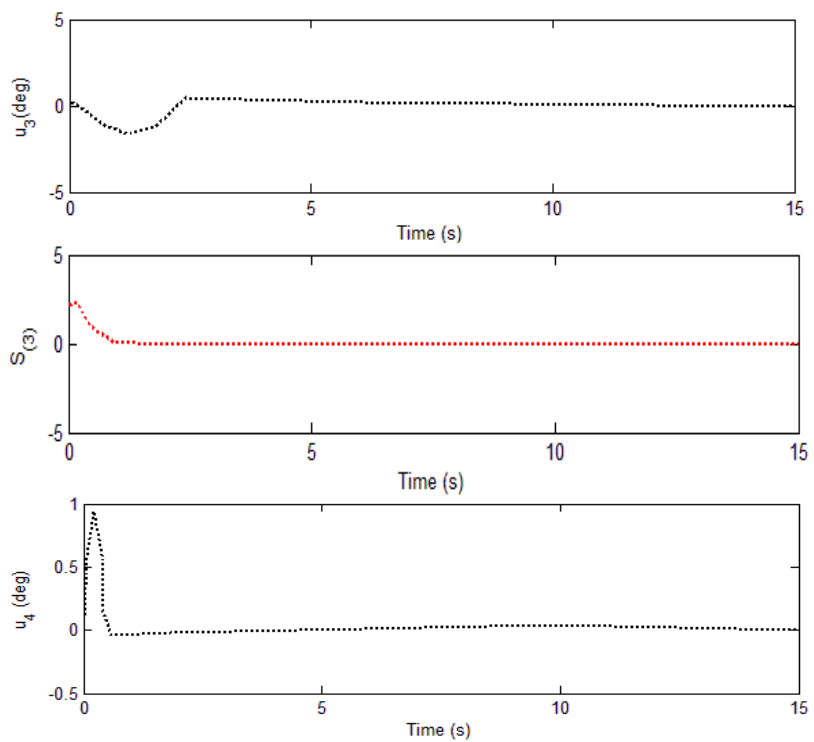

Figure 8. Sliding Variables

Case II: In this case of simulation, to validate the robustness of the ASMC method, the altitude and attitude tracking of UAV is performed. The simulations are performed, which is shown in Figure 9 to 14, that illustrates that ASMC will fulfill the control commands of input to reach at the reference position in a reasonable interval of time. The starting position and angles of the tri-rotor UAV for this simulation tests are $[0,0,0] \mathrm{m}$ and $[0,0$, $0] \mathrm{rad}$. The reference position and Euler angles are used for the simulation tests are: [10, $10,10] \mathrm{m}$ and $[0,0,0] \mathrm{rad}$ which is shown in Figure 9 and 10.

Figure 11 and 12 shows the linear and angular velocity subcomponent which displays the similar behavior as the corresponding altitude and attitude angles. Definitely, these 
state variables are driven to their steady states as estimated and once again, results demonstrates the effectiveness of the ASMC.

Moreover, the controller " $u_{1}$ " reaches its reference state at about 3 seconds and proves that the matrix $\mathrm{Q}$ is time invariant. In Figure 13, all the input control commands are shown, which are followed by the sliding variables of the controller which are $S_{1}, S_{2}$ and $S_{3}$ that converges to zero at a finite interval of time and is shown in Figure 14. Furthermore, control input $u_{1}=0$, proves that "Q" matrix is time invariant, at the start $u_{1}$ to $u_{4}$ have high oscillations, but it will converge to zero at a fair amount of time.

(Wind disturbance test): When the aircraft starts at the initial position, the disturbance of wind is along with it, in the direction of flight that will deviate the flight reference path. In the worst case scenario, the deviation of a reference path in the real world will directly affect the failure of flight. That's why in this article, a wind disturbance test is done to the check the performance. For this wind disturbance test, initially a wind is created artificially, in which the generated wind velocity polynomial is described as follows.

$$
W_{V}=-1.1299 x^{5}+4.25 x^{4}-.622 x^{\mathrm{a}}+3.45 x^{2}-2.17 x+3.11
$$

To check the efficiency of the proposed controller along with external wind disturbance, an external velocity of wind is taken for the $\mathrm{x}$-axis direction of UAV which is $1.25 \mathrm{~m} / \mathrm{sec}$, however, the roll angle of the aircraft change to 12.5 degree to hovering state. Furthermore, the gravity " $g_{e}$ " is same as hovering state " $u_{x}$ ". Thus, wind disturbance is applied to the tri-rotor roll angle in the direction of UAV along $\mathrm{x}$-axis,

$$
\tan \varphi=g_{s} / u_{x}
$$

Same, as the wind disturbance in y-direction change the angle of pitch with the same degree. Moreover, tri-rotor focuses to slow time changing aerodynamic interface and noises. To check the validity of the proposed controller nonlinearity, noises and wind disturbance use as a constraint, in which the wind disturbance to the aircraft are as follows,

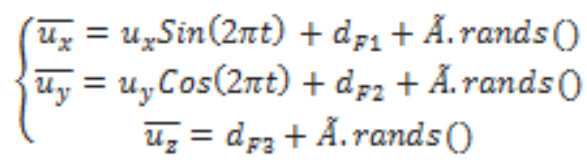

Where ' $\overline{u_{x}}$ ", ' $\overline{u_{y}}$ " and ' $\overrightarrow{u_{z}}$ ' are the wind disturbance external forces along with the nonlinearity applied to the aerial vehicle in the direction of $\mathrm{x}, \mathrm{y}$, and $\mathrm{z}$ axes respectively, " $u_{x}$ " and " $u_{y}$ " are the constant components of wind disturbance. The magnitude of random noise is denoted as " $\hat{A}$ " and the time varying interface force is $\mathrm{d}^{\prime}(x, t)=\left[d_{F 1}, d_{F 2}, d_{F 3}\right]$. The external wind disturbance is applied to the aircraft are $u_{x}=0.75 \mathrm{~N}$ and $u_{y}=0.75 \mathrm{~N}$ respectively. Now rewrite the aerodynamic interface force which is $\mathrm{d}^{\prime}(x, t)=[0.1 \operatorname{Sin}(0.1 \pi t), 0.1 \operatorname{Cos}(0.1 \pi t), 0.1 \operatorname{Cos}(0.1 \pi t)]$, and $\tilde{A}=0.19$. 

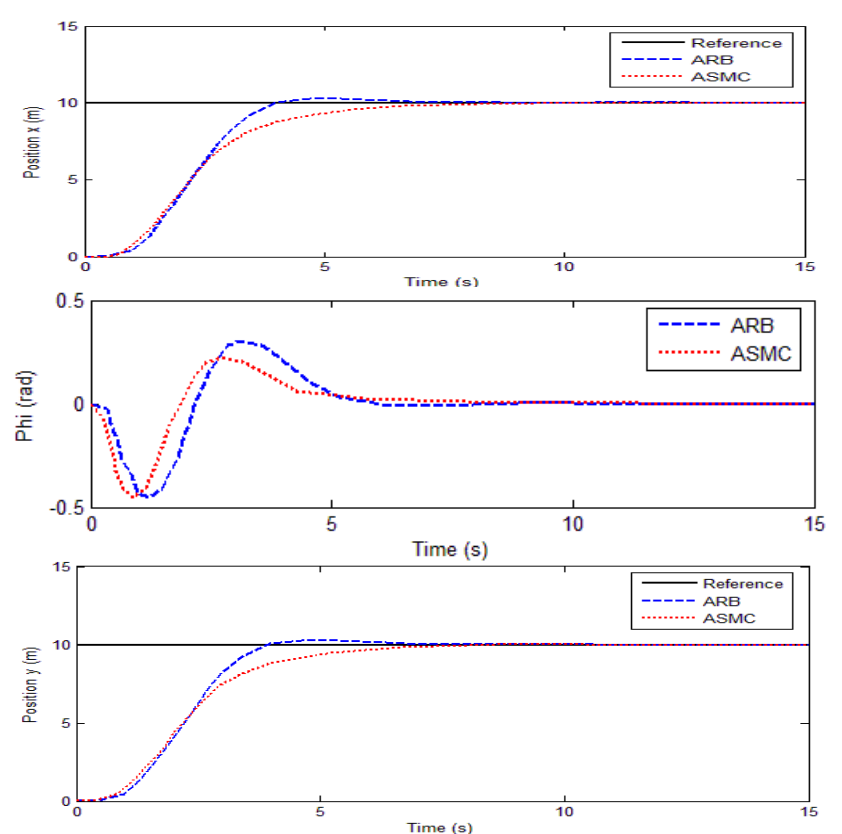

Figure 9. Positions $(x, y, z)$ of UAV
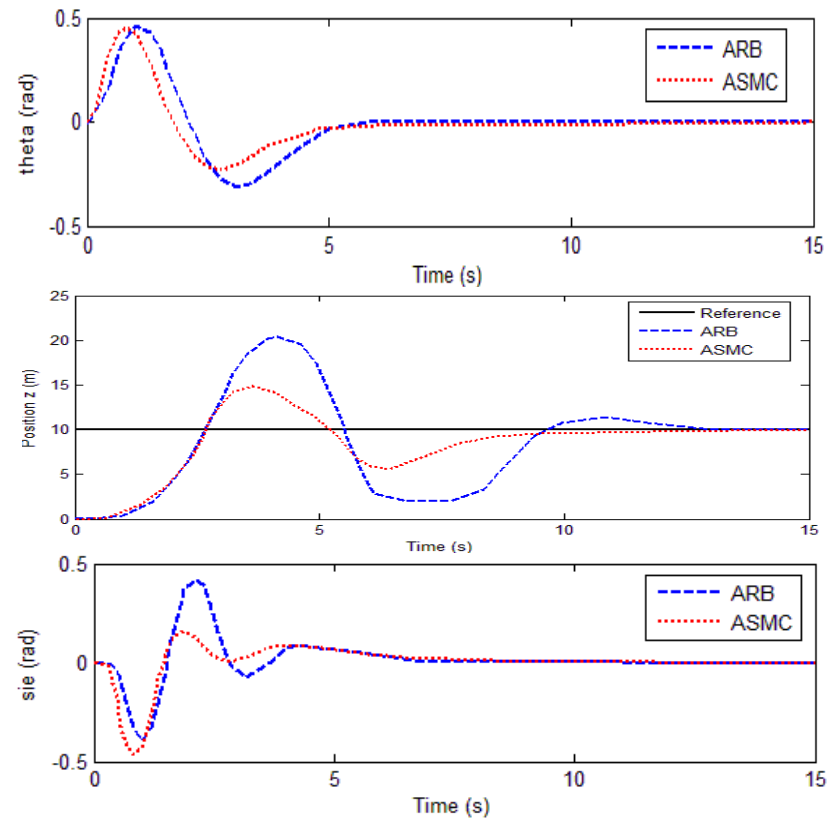

Figure 10. The Euler Angle Responses 

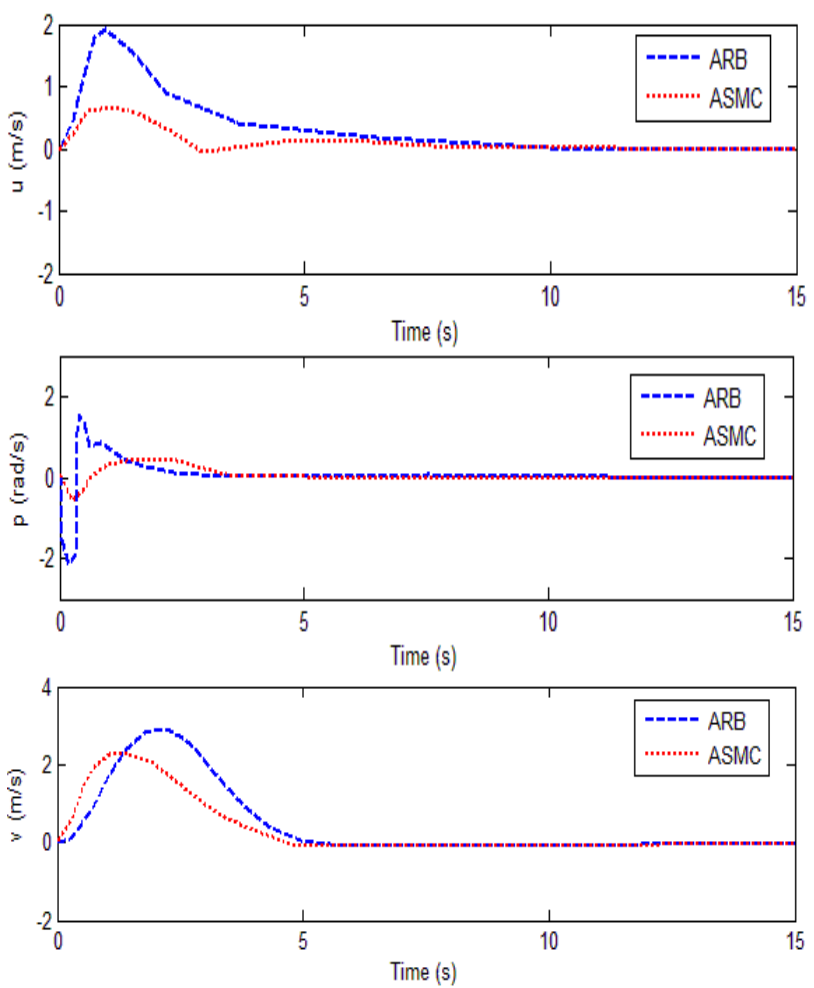

Figure 11. Linear Velocity Components
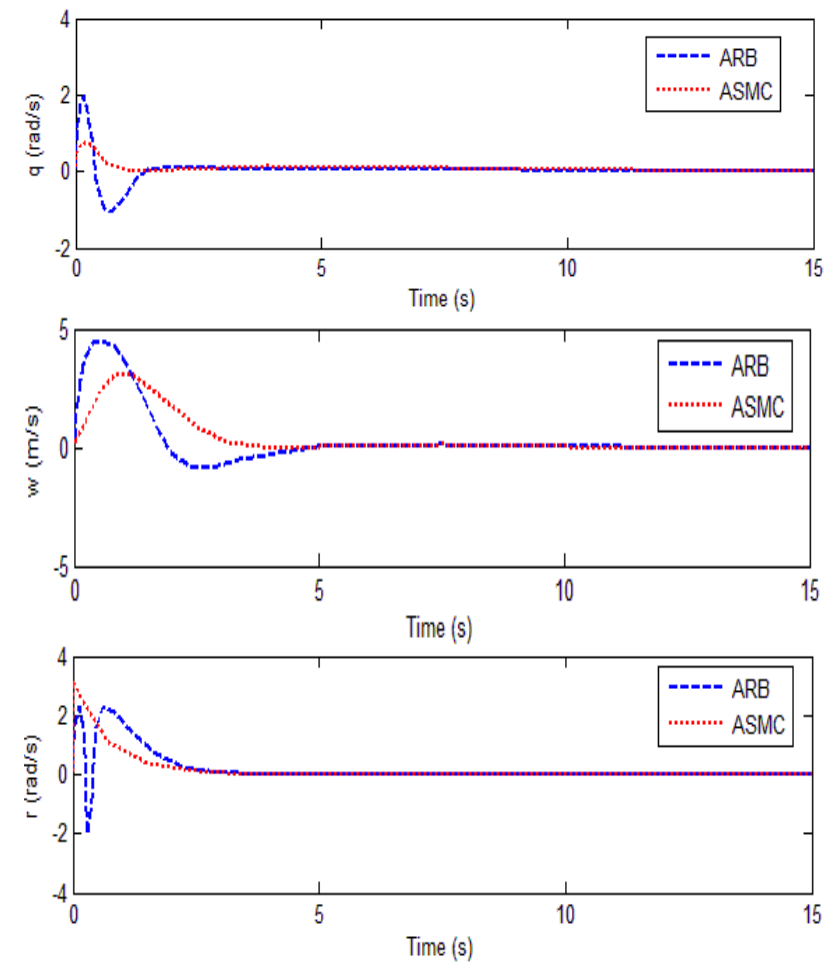

Figure 12. Angular Velocity Components 

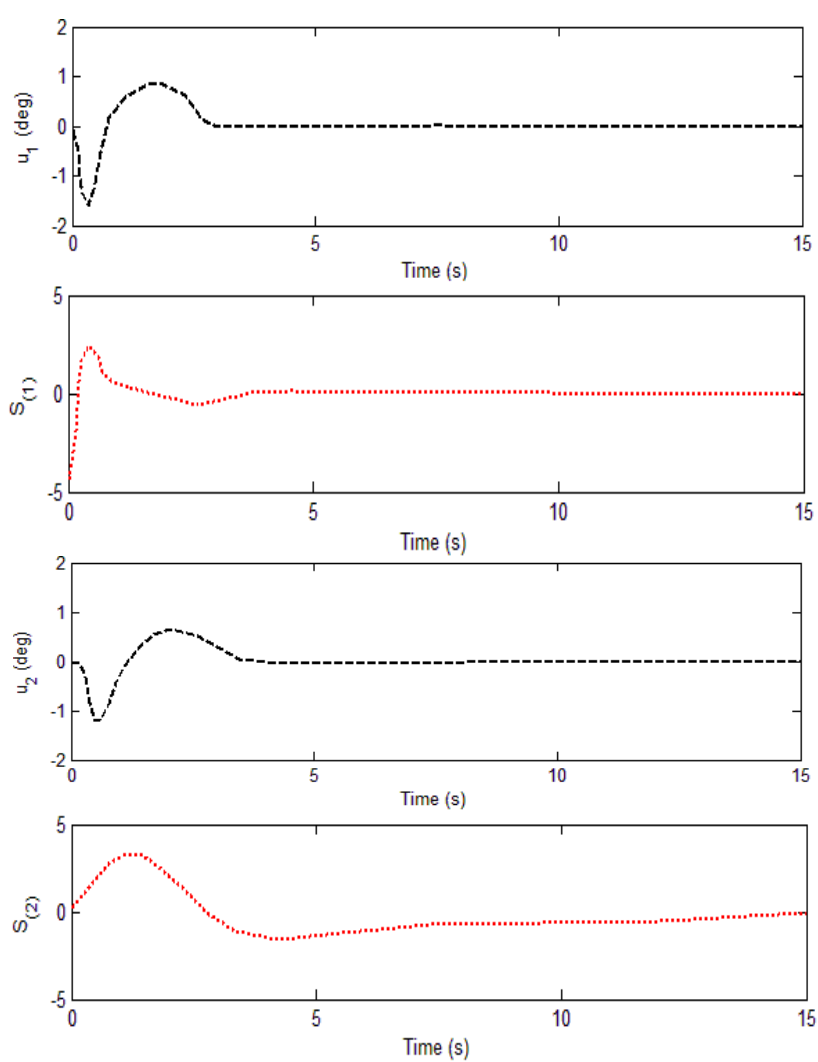

Figure 13. Input Control Commands
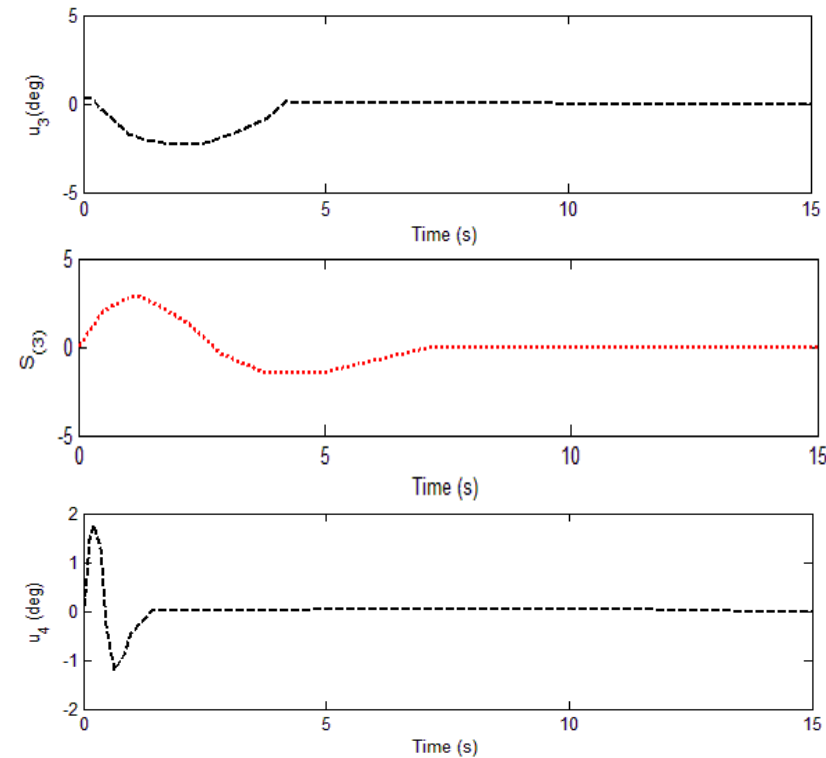

Figure 14. Sliding Variables

\section{Conclusion}

In the presented work, the ASMC is used for controlling the dynamics and stabilization of the altitude and attitude of tri-rotor aerial vehicle along with position control. The proposed system is simulated with two conditions, first ARB and ASMC comparison is given without disturbance and the controller successfully track the desired path. Secondly, 
the wind disturbance is introduced in the system such that the proposed controller offers better performance than ARB.

\section{Acknowledgement}

This work is sponsored by the Higher Education Commission (H.E.C) of Pakistan.

\section{References}

[1] P. W. Singer, "Wired for war: The robotics revolution and conflict in the 21st century", Penguin, (2009).

[2] R. H. Stone and G. Clarke, "The T-wing: a VTOL UAV for defense and civilian applications", University of Sydney, (2001).

[3] U. Ozdemir, Y. O. Aktas, A. Vuruskan, Y. Dereli, A. F. Tarhan, K. Demirbag, A. Erdem, G. D. Kalaycioglu, I. Ozkol and G. Inalhan, "Design of a commercial hybrid VTOL UAV system", Journal of Intelligent \& Robotic Systems, vol. 74, no. 1-2, (2014), pp. 371-393.

[4] F. A. Smith, "Apparatus and method for vertical take-off and landing aircraft", (2011).

[5] I. Colomina and P. Molina, "Unmanned aerial systems for photogrammetry and remote sensing: A review", ISPRS Journal of Photogrammetry and Remote Sensing, vol. 92, (2014), pp. 79-97.

[6] C. R. Calabrese and Legislative Counsel, "The Future of Unmanned Aviation in the US Economy: Safety and Privacy Considerations", American Civil Liberties Union, (2014).

[7] J. Wu, Z.-K. Dong, Z.-G. Liu and G.-Q. Zhou, "Geo-registration and mosaic of UAV video for quickresponse to forest fire disaster", In International Symposium on Multispectral Image Processing and Pattern Recognition, International Society for Optics and Photonics, (2007).

[8] G. C. H. E. de Croon, M. Perçin, B. D. W. Remes, R. Ruijsink and C. De Wagter, "Introduction to Autonomous Flight", In The DelFly, Springer Netherlands, (2016), pp. 125-138.

[9] Z. A. Ali, D.-B. Wang and M. Aamir, "Fuzzy-Based Hybrid Control Algorithm for the Stabilization of a Tri-Rotor UAV", Sensors, vol. 16, no. 5, (2016), p. 652.

[10] J. Awrejcewicz, "Modeling, Simulation and Control of Nonlinear Engineering Dynamical Systems", Springer, (2009).

[11] J. Ratti, "QV: the quad winged, energy efficient, six degree of freedom capable micro aerial vehicle", (2011).

[12] D.-W. Yoo, H.-D. Oh, D.-Y. Won and M.-J. Tahk, "Dynamic modeling and stabilization techniques for tri-rotor unmanned aerial vehicles", International Journal Aeronautical and Space Sciences, vol. 11, no. 3, (2010), pp. 167-174.

[13] A. Mokhtari, A. Benallegue and Y. Orlov, "Exact linearization and sliding mode observer for a quadrotor unmanned aerial vehicle", International Journal of Robotics \& Automation, vol. 21, no. 1, (2006), p. 39.

[14] H.-B. Duan and P. Li, "Progress in control approaches for hypersonic vehicle", Science China Technological Sciences, vol. 55, no. 10, (2012), pp. 2965-2970.

[15] Z. T. Dydek, A. M. Annaswamy and E. Lavretsky, "Adaptive control of quadrotor UAVs: A design trade study with flight evaluations", IEEE Transactions on control systems technology, vol. 21, no. 4, (2013), pp. 1400-1406.

[16] H.-Y. Chao, Y. Luo, L. Di and Y. Q. Chen, "Roll-channel fractional order controller design for a small fixed-wing unmanned aerial vehicle", Control Engineering Practice, vol. 18, no. 7, (2010), pp. 761-772.

[17] T. Hamel and R,. Mahony, "Visual servoing of an under-actuated dynamic rigid-body system: an imagebased approach", IEEE Transactions on Robotics and Automation, vol. 18, no. 2, (2002), pp. 187-198.

[18] L. Besnard, Y. B. Shtessel and B. Landrum, "Quadrotor vehicle control via sliding mode controller driven by sliding mode disturbance observer", Journal of the Franklin Institute, vol. 349, no. 2, (2012), pp. 658-684.

[19] W. Chen, B.-F. Song and N.-H. Meyer, "Adaptive Robust Backstepping (ARB) Control for Quadrotor Robot in Presence of Payload Variation and Unknown Disturbances", International Journal of Control and Automation vol. 9, no. 3, (2016), pp. 417-434.

[20] Z. A. Ali, D.-B. Wang and M. Aamir, "Fuzzy-Based Hybrid Control Algorithm for the Stabilization of a Tri-Rotor UAV", Sensors, vol. 16, no. 5, (2016), pp. 652.

[21] Z. A. Ali, D.-B. Wang, S. Masroor and M. Shafiq, "Attitude and Altitude Control of Tri-Rotor UAV by using Adaptive Hybrid Controller".

[22] Z. T. Dydek, A. M. Annaswamy and E. Lavretsky, "Adaptive control of quadrotor UAVs in the presence of actuator uncertainties", AIAA Infotech@ Aerospace, (2010), pp. 20-22.

[23] B. L. Stevens, F. L. Lewis and E. N. Johnson, "Aircraft Control and Simulation: Dynamics, Controls Design, and Autonomous Systems", John Wiley \& Sons, (2015).

[24] T. A. Ducan, "VTOL aircraft control method", (1995).

[25] J. A. Fax and R. M. Murray, "Information flow and cooperative control of vehicle formations", IEEE transactions on automatic control, vol. 49, no. 9, (2004), pp. 1465-1476. 
[26] A. Sanchez, L. R. G. Carrillo, E. Rondon, R. Lozano and O. Garcia, "Hovering flight improvement of a quad-rotor mini UAV using brushless DC motors", Journal of Intelligent \& Robotic Systems, vol. 61, no. 1-4, (2011), pp. 85-101.

[27] M. Wierema, "Design, implementation and flight test of indoor navigation and control system for a quadrotor UAV", Master of Science in Aerospace Engineering at Delft University of Technology, (2008).

[28] Y. Naidoo, R. Stopforth and G. Bright, "Quad-rotor unmanned aerial vehicle helicopter modelling \& control", International Journal of Advanced Robotic Systems, vol. 8, no. 4, (2011), pp. 139-149.

[29] L. B. Xu, S. X. Yang and B. Mo, "Pitching Dynamic Response of Variable Sweep Wing Aircraft", In Applied Mechanics and Materials, vol. 197, (2012), pp. 159-163. Trans Tech Publications.

[30] J. A. Carretero, R. P. Podhorodeski, M. A. Nahon and C. M. Gosselin, "Kinematic analysis and optimization of a new three degree-of-freedom spatial parallel manipulator", Journal of mechanical design, vol. 122, no. 1 (2000), pp. 17-24.

[31] H. Seydoux, M. Lefebure, F. Callou, C. Jonchery and L. Jean-Baptiste, "Method of piloting a rotarywing drone with automatic stabilization of hovering flight", (2009).

[32] J. Paulos and M. Yim, "Flight performance of a swashplateless micro air vehicle", IEEE International Conference on Robotics and Automation (ICRA), IEEE, (2015).

[33] P. C. Garcia, R. Lozano and A. E. Dzul, "Modelling and control of mini-flying machines", Springer Science \& Business Media, (2006).

[34] A. Askari, M. Mortazavi and H. A. Talebi, "UAV formation control via the virtual structure approach", Journal of Aerospace Engineering, vol. 28, no. 1, (2013).

[35] X.-D. Zhang, X.-L. Li, K. Wang and Y.-J. Lu, "A survey of modelling and identification of quadrotor robot", In Abstract and Applied Analysis, Hindawi Publishing Corporation, (2014).

[36] R. E. Maine and K. W. Iliff, "Application of parameter estimation to aircraft stability and control: The output-error approach", (1986).

[37] D. Gebre-Egziabher, G. H. Elkaim, J. D. Powell and B. W. Parkinson, "A gyro-free quaternion-based attitude determination system suitable for implementation using low cost sensors", In Position Location and Navigation Symposium, IEEE, (2000). 
International Journal of Control and Automation Vol.10, No.3 (2017) 